\title{
Can yeast isolation be predicted in complicated secondary non-postoperative intra-abdominal infections?
}

Hervé Dupont ${ }^{1,2^{*}}$, Mathieu Guilbart ${ }^{1}$, Alexandre Ntouba ${ }^{1}$, Mélanie Perquin' ${ }^{1}$ Sandra Petiot ${ }^{1}$, Jean-Marc Regimbeau ${ }^{3}$, Taieb Chouaki ${ }^{4}$, Yazine Mahjoub ${ }^{1,2}$ and Elie Zogheib ${ }^{1,2}$

\begin{abstract}
Introduction: The aim of this study was to create a predictive score for yeast isolation in patients with complicated non-postoperative intra-abdominal infections (CNPIAI) and to evaluate the impact of yeast isolation on outcome.

Methods: All patients with a CNPIAI undergoing emergency surgery over a three-year period were included in the retrospective cohort $(R C, n=290)$. Patients with a yeast-positive peritoneal fluid culture (YP) were compared with patients with a yeast-negative culture (YN). Multivariate logistic regression was used to identify factors independently associated with yeast isolation and a predictive score was built. The score's performance was then established in the prospective cohort $(P C, n=152)$ over an 18-month period. Outcome of the whole cohort was evaluated and independent risks factors of mortality searched.
\end{abstract}

Results: In the RC, 39 patients (13.4\%) were YP. Four factors were independently associated with the YP group: length of stay before surgery $\geq 48 \mathrm{~h}$ (odds ratio (OR) (95\% confidence interval $(C I))=3.1$ (1.4 to 6.9), $P=0.004,1$ point), per-operative cardiovascular failure (2.4 (1.1 to 5.8), $P=0.04,1$ point), generalized peritonitis (6.8 (2.7 to 16.7$), P<0.001,2$ points) and upper gastrointestinal tract perforation (2.5 (1.2 to 5.6), $P=0.02$, 1 point). In the $P C$, the area under the curve (95\%Cl) of the predictive score's receiver operating characteristic curve was 0.79 (0.72 to 0.86 ). For predicting an intra-abdominal candidiasis (IAC), a score $\geq 3$ had a sensitivity of 0.60 , a specificity of 0.84 , a positive predictive value of 0.49 and a negative predictive value of 0.89 . Furthermore, yeast isolation was associated with worse outcome and independently associated with mortality in the whole cohort $(\mathrm{OR}=2.15 ; 95 \% \mathrm{Cl}(1.03$ to 4.46$), P=0.04)$.

Conclusions: The new predictive score can be used to rule out intra-abdominal candidiasis and thus avoid the initiation of antifungal treatment. It is suited to less severe infections than previously published scores. IAC is associated independently with an increased mortality in this population.

\section{Introduction}

The pathogenicity of yeasts isolated in the peritoneal fluid of patients with complicated intra-abdominal infections (and especially community-acquired infections (CAIs)) is subject to debate. There are some data to suggest that yeasts have an impact on the outcome in cases of peptic ulcer perforation [1]. Non-postoperative nosocomial intra-abdominal infections (NPNIAIs) share certain

\footnotetext{
* Correspondence: dupont.herve@chu-amiens.fr

'Department of Anesthesiology and Critical Care Medicine, Amiens University Medical Center, 80054 Amiens, Cedex 1, France

2INSERM U1088, Jules Verne University of Picardy, Chemin du Thil, 80039

Amiens, Cedex 1, France

Full list of author information is available at the end of the article
}

microbiological characteristics with CAIs [2], such as the frequency of yeast isolation in peritoneal fluid samples. In view of the high mortality rate observed in patients with post-operative infections [3] and patients with organ failure admitted to the intensive care unit (ICU) [4], the pathogenicity of yeast in these contexts has been investigated more. However, the guidelines on the management of these types of infection are essentially based on extrapolation of data on candidemia $[5,6]$. Even though yeast intra-abdominal infections can be candidemic [7], the frequency is low and the diseases do not have the same course [8]. A number of scores have been developed in order to predict the occurrence of candidemia in high-risk patients, including 
the colonization index [9], Leon et al.'s Candida score [10] and a clinical rule [11]. However, none of these scores is suitable for complicated intra-abdominal infections. Furthermore, each of these scores has a high negative predictive value (NPV, for ruling out yeast infection) rather than a high positive predictive value (PPV, for initiating treatment) [12]. Ten years ago, Dupont et al. developed a score for severe complicated intra-abdominal infections in the ICU [13]. This is still the only available score with a moderately good PPV and NPV [13]. However, it was developed in a severe population of ICU patients. There are few data on less severe patients having undergone emergency surgery. The objectives of the present study were to (i) build a predictive score for yeast isolation in the peritoneal fluid of patients with complicated non-postoperative intra-abdominal infections (CNPIAI) in a retrospective cohort of patients and (ii) validate the score in a separate prospective cohort. The new score will be compared with previously described scores. The relationship between intra-abdominal candidiasis (IAC) and the outcomes for patients with complicated CNPNIAIs was also evaluated.

\section{Material and methods}

\section{Study design and patients}

This study was conducted in three parts. In the first part (score construction), patients with a CNPIAI and having undergone emergency surgery in our tertiary university hospital were retrospectively included over a three-year period (from January 2009 to December 2011). Patients with yeast-positive (YP) peritoneal fluid culture were compared with patient with a yeast-negative $(\mathrm{YN})$ culture. A predictive score was built by taking account of factors independently associated with IAC in the cohort. In the second part of the study (score validation), patients were included in a prospective cohort over an 18-month period (from January 2012 to June 2013). The performance of the new score was compared with previously published predictive scores (Dupont et al. [13], Leon at al. [10] and Paphitou et al. [14]). In the third part, the impact of IAC on outcome in the whole cohort was assessed.

The study's objectives and procedures were approved by the local investigational review board (Commission d'Evaluation Ethique de la Recherche Non Interventionnelle, Amiens, France). In accordance with French legislation, the need for informed consent was waived because of the observational nature of the study.

Patients with infected acute pancreatitis, postoperative nosocomial infections, acute trauma perforation $<6$ hours and primary peritonitis (such as infected ascites) were not included in the study.

\section{Surgery and microbiological management}

Surgery was performed by an experienced, trained team in accordance with the above-mentioned guidelines for the treatment of complicated intra-abdominal infections [15]. The definition of complicated intra-abdominal infection used is that presented in the Infectious Diseases Society of America (IDSA) guidelines: 'Complicated intraabdominal infection extends beyond the hollow viscus of origin into the peritoneal space and is associated with either abscess formation or peritonitis' [6]. Either laparoscopy or laparotomy was performed (depending on the diagnosis and the surgeon's choice). All peritoneal fluid samples were sent for microbiological and mycological testing. Antimicrobial therapy was initiated as soon as possible and in accordance with local treatment protocols. Treatment of yeast infection was only considered if the patient had organ failure. At least one preoperative blood culture was available for each patient. The mycology department performed yeast cultures and susceptibility testing. A strain of Candida was considered resistant to fluconazole for a minimum inhibitory concentration $>32 \mu \mathrm{g} \cdot \mathrm{ml}^{-1}$.

\section{Definitions and data collection}

The etiology of the intra-abdominal infection, the extent of the infection (generalized or localized) and the perforation site were recorded. The hospital length of stay before surgery was noted. Demographic data and the underlying disease were noted from the patient's medical records. A number of severity scores were calculated: the American Society of Anesthesiologist score [16], the Mannheim peritonitis index [17], the Acute Physiology and Chronic Health Evaluation II (APACHE II) score [18], the Simplified Acute Physiology Score II (SAPS II) [19] and the Sepsis-related Organ Failure Assessment (SOFA) score [20]. Cardiovascular failure was defined by the need for norepinephrine during surgery (despite fluid challenge). Respiratory failure was defined by the need for more than 24 hours of mechanical ventilation. The ICU admission rate, lengths of stay (in the ICU or a hospital ward) and in-hospital mortality were assessed.

\section{Statistical analysis}

Results are expressed as mean \pm standard deviation (SD) or number (proportion). First, patients in the YP group were compared with those in the YN group via a chisquared test (with Yates' correction, if necessary) for qualitative variables and a two-sided $t$ test for quantitative variables. A multivariate stepwise logistic regression model (backward Wald model) was built in order to identify any independent factors for yeast isolation in patients with intra-abdominal infections. Only significant variables $(P<0.05)$ in a univariate analysis were included in the multivariate model. All potential explanatory variables included in the multivariate analyses were subjected to a collinearity analysis in a correlation matrix. Intercorrelated variables were not included in the multivariate model (tolerance $<0.3$ and variance inflation factor $>3$ ). 
Adjusted odds ratios (ORs) and their 95\% confidence intervals $(95 \% \mathrm{CIs})$ are reported. The constant (intercept) was only included in the model when statistically significant [21]. The Hosmer-Lemeshow test was used to assess the model's goodness of fit [21]. The statistical significance of individual regression coefficients was assessed with the Wald chi-squared test [21]. The model's predicted probabilities were validated with the $c$ statistic (corresponding to the model's area under the curve (AUC)) [21].

A score was built according to the ORs in the multivariate analysis and was tested in a receiver operating characteristic (ROC) analysis [22]. The best score's cutoff was calculated for the best Youden index. The score's performance was tested in a prospective (validation) cohort, according to the same analysis. The AUC of the ROC curve of new score was compared with the AUC of previously published scores in the same cohort using Hanley and McNeil tests.

A second multivariate analysis was performed to identify the impact of yeast isolation in mortality of the whole cohort. The threshold for statistical significance was set to $P \leq 0.05$. Statistical analyses were performed with PASW Statistics 18 software (IBM Inc., Chicago, IL, USA) and MedCalc 12.7.5 software (MedCalc Software, Ostend, Belgium).

\section{Results}

Four hundred and forty-four patients were included in the study (290 in the retrospective cohort and 152 in the prospective cohort). Location of perforation, main etiologies and microbiological cultures of the peritoneal fluid are exposed in Tables 1 and 2. Bacteremia occurred in $9.3 \%$ of the patients and no candidemia was observed.

\section{Predictive factors for yeast isolation}

Two hundred and ninety patients were included in the retrospective cohort. Of these, 39 (13.4\%) had an intraabdominal candidiasis ( $72.5 \%$ of Candida albicans). Fifteen percent of the strains were fluconazole-resistant. The demographic characteristics of the $\mathrm{YP}$ and $\mathrm{YN}$ groups are summarized in Table 3. There were no significant intergroup differences other than a higher proportion of patients on immunosuppression in the YP group $(P=0.006)$. In all, $74.1 \%$ of the patients had a CAI; these were essentially gallbladder perforations $(n=56,19.3 \%)$, appendix perforations $(n=90,31.1 \%)$ and colon perforations $(n=$ 81, 27.9\%). There were marked intergroup differences in the severity scores and types of infection (Table 3). Patients in the YP group were significantly more likely to have worse severity scores $(P<0.001$ for all $)$, NPNIAIs $(P<0.001)$, generalized infections $(<0.001)$, upper gastrointestinal tract perforation $(P<0.001)$ and ongoing antimicrobial therapy ( $\geq 48$ hours) $(P=0.01)$. The results of the multivariate analysis are reported in Table 4. Four
Table 1 Location and etiologies of complicated non-postoperative intra-abdominal infections in the whole cohort of patients

\begin{tabular}{ll}
\hline & $\begin{array}{l}\text { Whole cohort } \\
(\mathbf{n}=\mathbf{4 4 2 )}\end{array}$ \\
\hline Lower gastrointestinal tract & $\mathbf{3 1 2 ( 7 0 . 6 )}$ \\
Appendicitis & $133(30.1)$ \\
Diverticulitis & $75(17)$ \\
Inflammatory bowel disease & $10(2.3)$ \\
Malignancy & $18(4.1)$ \\
Ischemic & $39(8.8)$ \\
Miscellaneous & $37(8.4)$ \\
Upper intestinal tract & $\mathbf{1 3 0 ( 2 9 . 4 )}$ \\
Biliary tract & $76(17.2)$ \\
Ulcus disease & $43(9.7)$ \\
Ischemic & $6(1.4)$ \\
Miscellaneous & $5(1.0)$ \\
\hline
\end{tabular}

Results are expressed as the number (proportion, in \%).

Table 2 Results of the peritoneal fluid cultures in the whole cohort of patients with complicated nonpostoperative intra-abdominal infections $(n=442)$

\begin{tabular}{cl}
\hline & $\begin{array}{l}\text { Isolates } \\
\text { ( } \mathbf{n}=\mathbf{9 2 7})\end{array}$ \\
\hline Aerobes & $\mathbf{6 7 7}(73.0)$ \\
Gram-negative bacilli & $\mathbf{4 2 6}(45.9)$ \\
Escherichia coli & 270 \\
Klebsiella spp. & 43 \\
Enterobacter spp. & 12 \\
Non-fermenting bacilli & 30 \\
Miscellaneous & 71 \\
Gram-positive cocci & $\mathbf{2 5 1}(27.1)$ \\
Streptococci & 109 \\
Staphylococci & 14 \\
Enterococci & 128 \\
Anaerobes & $\mathbf{1 7 8}(19.2)$ \\
Bacteroides spp. & 138 \\
Clostridium spp. & 18 \\
Miscellaneous & 22 \\
Fungi* & $\mathbf{7 2}(7.7)$ \\
Candida albicans & 47 \\
Candida glabrata & 8 \\
Candida tropicalis & 7 \\
Candida krusei & 3 \\
Miscellaneous & 7 \\
\hline
\end{tabular}

Results are expressed as the number (proportion, in \%). ${ }^{*}$ Thirteen patients had a pure culture of Candida ( $18.8 \%$ of the fungal infections). 
Table 3 Demographic data for the retrospective cohort according of the presence (yeast positive) or absence (yeast negative) of yeast in the peritoneal fluid culture

\begin{tabular}{|c|c|c|c|}
\hline & $\begin{array}{l}\text { Yeast positive } \\
(\mathrm{n}=39)\end{array}$ & $\begin{array}{l}\text { Yeast negative } \\
(n=251)\end{array}$ & $P$ value \\
\hline$\overline{\text { Age }}$ & $65 \pm 18$ & $59 \pm 22$ & 0.08 \\
\hline Female gender & $20(51.3)$ & $125(49.8)$ & 0.98 \\
\hline BMI $\left(\mathrm{kg} \cdot \mathrm{m}^{-2}\right)$ & $26.6 \pm 7.2$ & $25.9 \pm 5.7$ & 0.95 \\
\hline \multicolumn{4}{|l|}{ Underlying diseases } \\
\hline Prior abdominal surgery & $7(17.9)$ & $46(18.1)$ & 0.87 \\
\hline Diabetes & $7(17.9)$ & $40(15.9)$ & 0.93 \\
\hline Immunosuppression & $7(17.9)$ & $12(4.8)$ & 0.006 \\
\hline Chronic cardiovascular disease & $22(56.4)$ & $120(47.8)$ & 0.41 \\
\hline Chronic renal failure & $3(7.7)$ & $9(3.6)$ & 0.15 \\
\hline ASA status & $2.9 \pm 0.6$ & $2.5 \pm 0.8$ & 0.001 \\
\hline Mannheim peritonitis index score & $22.9 \pm 7.6$ & $16.0 \pm 8.1$ & $<0.001$ \\
\hline APACHE II score & $14.6 \pm 10.9$ & $8.0 \pm 7.9$ & $<0.001$ \\
\hline SAPS II score & $36.2 \pm 20.6$ & $24.7 \pm 15.2$ & $<0.001$ \\
\hline SOFA score & $4.9 \pm 6.5$ & $1.9 \pm 3$ & $<0.001$ \\
\hline \multicolumn{4}{|l|}{ Type of infection } \\
\hline Community-acquired & $19(48.7)$ & $196(78.1)$ & $<0.001$ \\
\hline LOS $\geq 48 \mathrm{~h}$ before surgery & $20(51.3)$ & $55(21.9)$ & \\
\hline Generalized peritonitis & $31(79.5)$ & $95(37.8)$ & $<0.001$ \\
\hline Upper gastrointestinal tract location & $18(46.2)$ & $74(29.6)$ & $<0.001$ \\
\hline Ongoing $A B \geq 48 \mathrm{~h}$ & $12(30.8)$ & 35 (13.9) & 0.01 \\
\hline
\end{tabular}

Results are expressed as the mean \pm standard deviation or the number (proportion, in \%). BMI, body mass index; ASA, American Society of Anesthesiology; APACHE II, Acute Physiology and Chronic Health Evaluation II; SAPS II, Simplified Acute Physiology Score II; SOFA, Sepsis-related Organ Failure Assessment; LOS, length of stay; $A B$, antimicrobial therapy.

independent factors were predictive of IAC: length of stay before surgery $\geq 48 \mathrm{~h}$, per-operative cardiovascular failure, generalized peritonitis and upper gastrointestinal tract perforation. The model's Wald chi-squared statistic was $50.2(\mathrm{df}=5, P<0.001)$. The Hosmer-Lemeshow test statistic was $9.69(\mathrm{df}=6, P=0.14)$. The model's $c$ statistic was 0.83 (0.77 to 0.89 ).

Table 4 Multivariate analysis of factors independently associated with an intra-abdominal candidiasis in the retrospective cohort with CAls and NPNIAls

\begin{tabular}{llll}
\hline Parameters & Adjusted OR & 95\%Cl & $\boldsymbol{P}$ value \\
\hline $\begin{array}{l}\text { Per-operative cardiovascular } \\
\text { failure }\end{array}$ & 2.43 & $1.01-5.81$ & 0.04 \\
$\begin{array}{l}\text { Upper gastrointestinal tract } \\
\text { perforation }\end{array}$ & 2.53 & $1.15-5.55$ & 0.02 \\
LOS $\geq 48$ h before surgery & 3.15 & $1.45-6.89$ & 0.004 \\
Generalized peritonitis & 6.78 & $2.75-16.68$ & $<0.001$ \\
\hline
\end{tabular}

CAls, community-acquired infections; NPNIAls, non-postoperative nosocomial intra-abdominal infections; $\mathrm{OR}$, odds ratio; $\mathrm{Cl}$, confidence interval; $\mathrm{LOS}$, length of stay.

\section{Construction and validation of the score}

A predictive score was built according to the ORs in the multivariate analysis (Table 5). In the retrospective cohort, the area $(95 \% \mathrm{CI})$ under the curve was 0.82 (0.73 to 0.90 ), yielding the following characteristics for a score $\geq 3$ : sensitivity $=0.75$; specificity $=0.77 ; \mathrm{PPV}=0.34 ; \mathrm{NPV}=0.95$; positive likelihood ratio $=3.2$; negative likelihood ratio $=0.34$.

The score was validated in a prospective cohort of 152 patients. There were no significant demographic or clinical differences between the retrospective and prospective cohorts (Table 6), except for a slightly higher SOFA score in

Table 5 Predictive score for intra-abdominal candidiasis in complicated non-postoperative intra-abdominal infections

\begin{tabular}{ll}
\hline Item & Value \\
\hline LOS $\geq 48 \mathrm{~h}$ before surgery & 1 point \\
Per-operative cardiovascular failure & 1 point \\
Generalized peritonitis & 2 points \\
Upper gastrointestinal tract perforation & 1 point
\end{tabular}

LOS, length of stay. 
Table 6 Comparison of the construction and validation cohorts

\begin{tabular}{llll}
\hline & $\begin{array}{l}\text { Construction cohort } \\
(\mathbf{n}=\mathbf{2 9 0})\end{array}$ & $\begin{array}{l}\text { Validation cohort } \\
(\mathbf{n}=\mathbf{1 5 2})\end{array}$ & $\boldsymbol{P}$ value \\
\hline Age & $60.2 \pm 21.7$ & $59.4 \pm 27.8$ & 0.74 \\
Female gender & $145(50.0)$ & $80(52.6)$ & 0.62 \\
BMI (kg.m $\left.{ }^{-2}\right)$ & $26.0 \pm 5.9$ & $26.5 \pm 16.8$ & 0.72 \\
Mannheim & $16.9 \pm 8.4$ & $17.8 \pm 4.2$ & 0.13 \\
peritonitis & & & \\
index score & & $2.5 \pm 1.4$ & 0.03 \\
SOFA score & $2.0 \pm 3.9$ & $9.3 \pm 7.1$ & 0.57 \\
APACHE II score & $8.9 \pm 8.6$ & $30(19.7)$ & 0.10 \\
IAC & $39(13.4)$ & $114(75.0)$ & 0.91 \\
Community- & $215(74.1)$ & & \\
acquired & & $57(37.5)$ & 0.29 \\
infection & & $20(13.2)$ & 0.43 \\
ICU admission & $93(32.1)$ & $30(10.3)$ &
\end{tabular}

Results are expressed as the mean \pm standard deviation or the number (proportion, in \%). BMI, body mass index; SOFA, Sepsis-related Organ Failure Assessment; APACHE II, Acute Physiology and Chronic Health Evaluation II; IAC, intra-abdominal candidiasis; ICU, intensive care unit.

the prospective cohort $(P=0.03)$. Thirty-two strains of yeasts were isolated, with essentially the same distribution as in the retrospective cohort (C. albicans: $62.5 \%)$ and the same proportion of fluconazole resistance (15.6\%). The proportion of YP samples as a function of the score is shown for each cohort in Figure 1. The score's AUC of the ROC curve for the prospective cohort was 0.79 (0.72 to 0.86 ). For a predictive score $\geq 3$, the sensitivity was 0.60 , the specificity was 0.84 , the PPV was 0.49 , the NPV was 0.90 , the positive likelihood ratio was 3.85 and the negative likelihood ratio was 0.47. The AUC of the ROC curve of the new score is presented in comparison with previously published scores of Dupont et al. [13], Leon et al. [10] and Paphitou et al. [14] in Figure 2. The new score had the best AUC when compared with other scores. However, no statistical differences were observed between the new score and Dupont's score $(P=0.15)$, the new score and Leon's score $(P=0.17)$. The former three scores were all significantly better than Paphitou's score $(P=0.0001 ; P=0.006 ; P=0.02$, respectively).

\section{Outcome of the whole cohort}

Main outcomes when comparing YP and YN groups are presented in Table 7. Briefly, patients with an IAC had more complications, had undergone more relaparotomies, and had more organ failure requiring ICU admission. Their hospital length of stay was twice as high and mortality three times higher $(27.5 \%$ vs. $8.3 \%, P<0.001)$. Univariate and multivariate analysis of mortality is exposed in Table 8. Four independent factors of mortality in the whole cohort were evidenced: an IAC, a Manheim peritonitis score $\geq 17$, an American Society of Anesthesiology (ASA) score $\geq 3$ and a SOFA score $\geq 1$. The model's Wald chi-squared statistic was $93.3(\mathrm{df}=4, P<0.001)$. The Hosmer-Lemeshow test statistic was $3.8(\mathrm{df}=6, P=0.694)$. The model's $c$ statistic was 0.79 (0.73 to 0.85 ). For the population of patients with IAC $(n=69), 52.2 \%$ were treated with an antifungal drug (39\% of azole and $61 \%$ of echinocandin). Mortality rate was $21.2 \%$ in the group treated versus $33.3 \%$ in the group not treated $(P=0.29)$.

\section{Discussion}

Our present results show that a YP peritoneal fluid culture is associated with worst outcomes and increased mortality in patients with CNPIAI. The prevalence of yeast isolation in this context is low (15.6\%). Four parameters were independently associated with IAC: length of

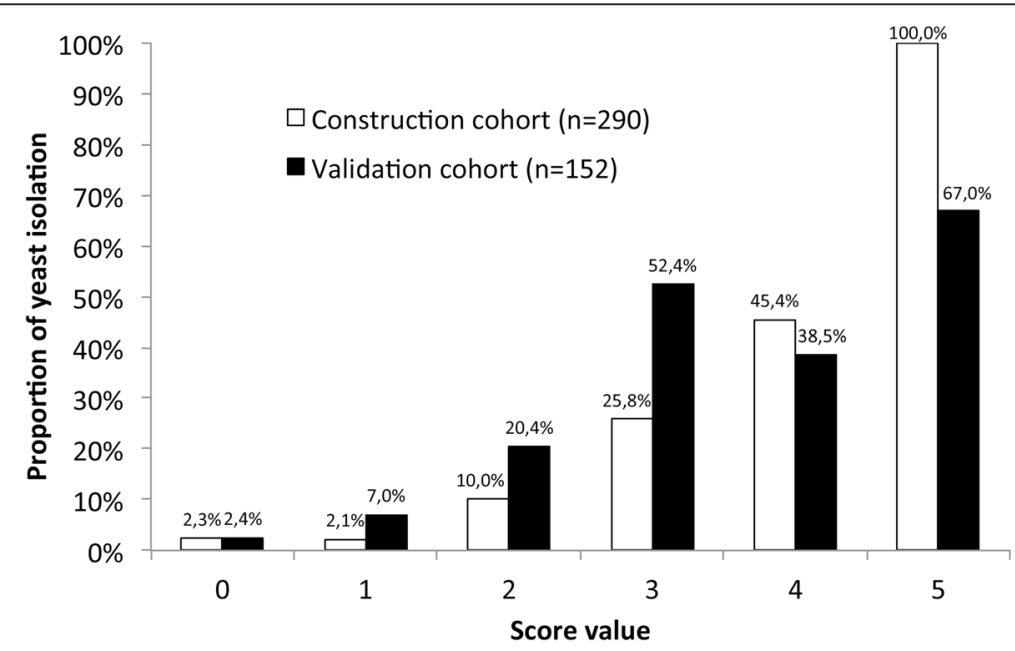

Figure 1 Comparison of the prevalence of a yeast-positive (YP) peritoneal fluid culture in the retrospective construction cohort ( $n=290$ ) and in the prospective validation cohort $(n=152)$, as a function of the score. 


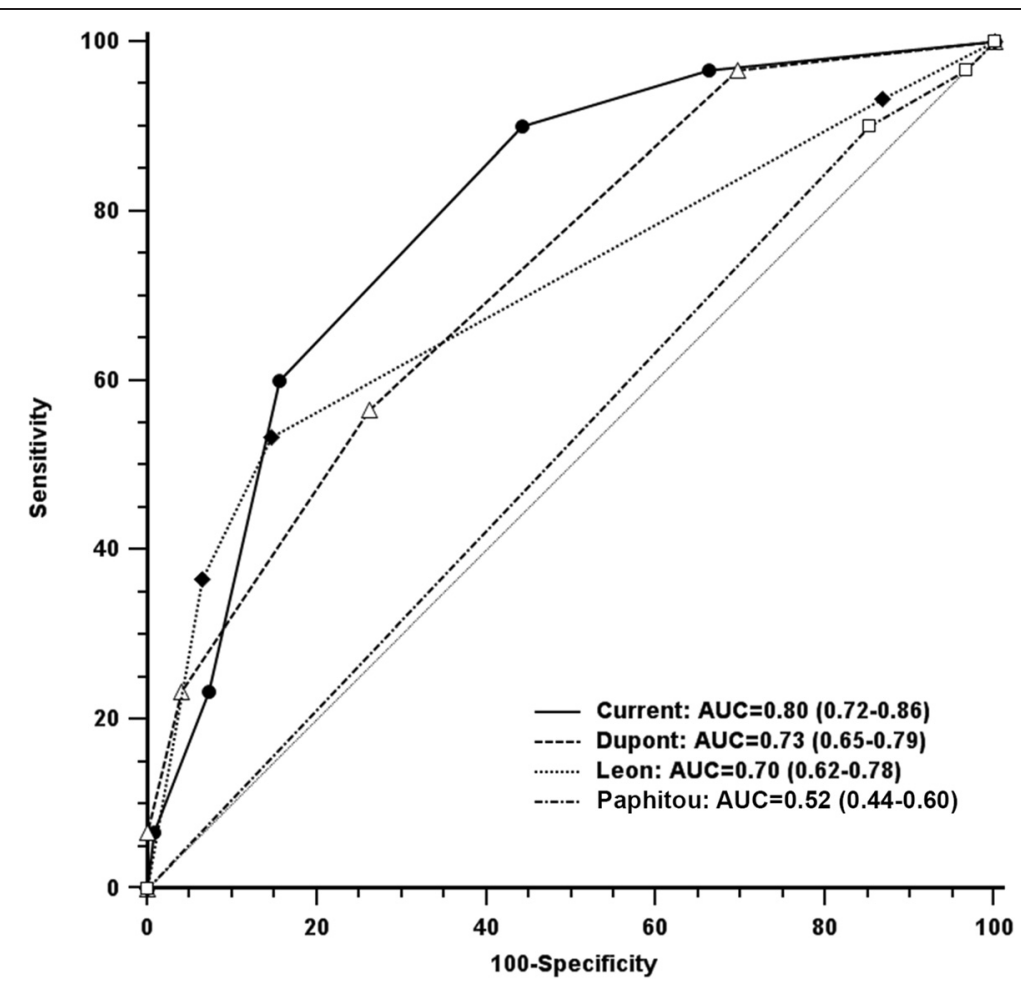

Figure 2 Comparison of receiver operating characteristic curves between the new score and previously published one in the prospective cohort (Dupont et al. [13], Leon et al. [10], Paphitou et al. [14]).

stay before surgery $\geq 48 \mathrm{~h}$, per-operative cardiovascular failure, generalized peritonitis and upper gastrointestinal tract perforation. The predictive score has a high NPV and thus can be used to rule out the presence of yeast in the peritoneal fluid. Hence, this score may constitute an easy- to-use bedside tool that enables the physician to avoid the initiation of inappropriate antifungal treatment.

Although yeasts are undoubtedly pathogenic in postoperative infections [3], this question is subject to debate in the context of CAIs and there are few data on this specific

Table 7 Comparison of outcomes between yeast-positive and yeast-negative groups with complicated non-postoperative intra-abdominal infections

\begin{tabular}{|c|c|c|c|}
\hline $\begin{array}{l}\text { Whole cohort } \\
(\mathrm{n}=442)\end{array}$ & $\begin{array}{l}\text { Yeast positive } \\
(n=69)\end{array}$ & $\begin{array}{l}\text { Yeast negative } \\
(n=373)\end{array}$ & $P$ value \\
\hline Any complication & $54(63.8)$ & $158(42.4)$ & 0.001 \\
\hline Infectious complication & $32(46.3)$ & $98(26.3)$ & 0.001 \\
\hline Digestive & 8 & 33 & \\
\hline Pneumonia & 12 & 27 & \\
\hline Miscellaneous & 12 & 38 & \\
\hline Transfusion & $19(27.5)$ & $38(10.2)$ & 0.001 \\
\hline Relaparotomy & $17(24.6)$ & $52(13.9)$ & 0.02 \\
\hline Cardiovascular failure & $36(52.2)$ & $64(17.2)$ & $<0.001$ \\
\hline Respiratory failure & $35(50.7)$ & $81(21.7)$ & $<0.001$ \\
\hline ICU admission & $39(56.5)$ & $111(29.8)$ & $<0.001$ \\
\hline Duration of mechanical ventilation (d) & $10.7 \pm 14.9$ & $9.5 \pm 14.7$ & 0.69 \\
\hline ICU length of stay (d) & $16.1 \pm 16.4$ & $11.6 \pm 13.9$ & 0.11 \\
\hline Hospital length of stay (d) & $20.5 \pm 22.4$ & $13.2 \pm 16.0$ & 0.001 \\
\hline Mortality & $19(27.5)$ & $31(8.3)$ & $<0.001$ \\
\hline
\end{tabular}

Results are expressed as the mean \pm standard deviation or the number (proportion, in \%). ICU, intensive care unit. 
Table 8 Predictive factors of mortality in the whole cohort of 442 patients with complicated non-postoperative intra-abdominal infections

\begin{tabular}{|c|c|c|c|c|c|c|}
\hline \multirow[t]{2}{*}{ Parameters } & \multicolumn{3}{|c|}{ Univariate analysis } & \multicolumn{3}{|c|}{ Multivariate analysis } \\
\hline & $\mathrm{OR}$ & $95 \% \mathrm{Cl}$ & $P$ value & AOR & $95 \% \mathrm{Cl}$ & $P$ value \\
\hline IAC & 4.19 & $2.20-7.98$ & 0.001 & 2.15 & $1.03-4.46$ & 0.04 \\
\hline Ongoing $A B \geq 48 h$ & 3.52 & $1.83-6.79$ & 0.001 & - & - & - \\
\hline MPI score $\geq 17$ & 7.96 & $3.31-19.10$ & 0.001 & 3.22 & $1.26-8.25$ & 0.02 \\
\hline ASA score $\geq 3$ & 19.42 & $5.95-63.47$ & 0.001 & 7.56 & $2.21-25.78$ & 0.001 \\
\hline SOFA score $\geq 1$ & 18.35 & $6.47-52.02$ & 0.001 & 7.90 & $2.68-23.26$ & 0.001 \\
\hline APACHE $\|$ score $\geq 7$ & 19.22 & $5.88-62.82$ & 0.001 & - & - & - \\
\hline
\end{tabular}

OR, odds ratio; $\mathrm{Cl}$, confidence interval; $\mathrm{AOR}$, adjusted odds ratio; IAC, intra-abdominal candidiasis; $\mathrm{AB}$, antimicrobial therapy; $\mathrm{MPI}$, Mannheim peritonitis index; $\mathrm{ASA}$, American Society of Anesthesiology; SOFA, Sepsis-related Organ Failure Assessment; APACHE II, Acute Physiology and Chronic Health Evaluation II.

topic. In a case-control study, there was no significant difference in outcome between YP and YN CAIs [3]. However, the study featured a small number of patients. In a study of patients with organ failure admitted to the ICU, a YP culture was found to be associated with elevated mortality [4]. Another study reported a significantly greater proportion of septic shock in CAIs when yeast was detected in the peritoneal fluid culture [23]. The overall prevalence of a YP culture in our study population (15.6\%) is similar to the mean value reported in the literature (with values ranging from $4 \%$ to $43.4 \%$ in studies of CAIs) $[2,24]$. The prevalence clearly depends on the study population in question. For example, the prevalence was very high in two studies that focused solely on peptic ulcer perforations [1,24]. Furthermore, yeast isolation was associated with increased morbidity and mortality in these studies $(21.7 \%$ vs. $3.4 \%$ [1] and $33.3 \%$ vs. $14.6 \%$ [24] for YP and YN patients, respectively).

In the present study, four parameters were found to be independently associated with IAC of patients with a CNPIAI. Interestingly, upper gastrointestinal tract perforation and per-operative cardiovascular failure were previously included in a predictive score for severe intraabdominal infections in the ICU [4]. Another Spanish study reported these two risk factors in 74 Candida peritonitis patients [25]. The two other parameters in the latter study (female gender and ongoing antimicrobial therapy for more than 48 hours) were not significant in our study [4]. However, ongoing antimicrobial therapy was associated with yeast isolation in our univariate analysis; this was probably due to the low prevalence of antimicrobial therapy because few patients had hospitalacquired infections. It has been well established that ongoing antimicrobial therapy is a recognized risk factor for candidemia [26]. However, in the present study, hospital length of stay $\geq 48 \mathrm{~h}$ before surgery was found to be independently associated with yeast isolation. There are no literature data on why yeast isolation is more frequently associated with generalized peritonitis than with localized infection. It could be only due to the major impact of appendicitis infections in CAI. The population investigated in the present study is mild to moderate with only a $33.9 \%$ rate of ICU admission and a low mortality of $11.3 \%$. It is very different to the Dupont et al. [13] or Leon et al. [10] studies with a $100 \%$ rate of ICU admission and reported mortality of $43 \%$ and $33 \%$, respectively.

The score developed in the present study has a good NPV. It may be important to avoid the inappropriate initiation of antifungal treatments that are costly and whose impact on resistance is not well known [27]. All the previously published scores or clinical rules were developed to predict the occurrence of candidemia (even in high-risk surgical patients) [9-11]. These scores have much the same operational values as our present score notably with very high NPV for avoiding treatment. The high NPV is essentially due to the low prevalence of the disease. However, the predictive value of a score has been shown to be better for severe intra-abdominal infections in the ICU than previously described for candidemia, with a PPV of $67 \%$ and a NPV of $72 \%$ [13]. The colonization index and the Candida score were recently tested for the prediction of blood culture-negative IAC but had very poor operational values [28]. Blood levels of $ß$-glucan may be of value for the diagnosis of postoperative infections in high-risk surgical patients [28]. However, there are no data on the value of $\beta$-glucan levels in CAIs. Furthermore, it may not be enough to know the change over time in $B$-glucan levels in CAIs because the physician has to decide at bedside whether antifungal treatment must be initiated or not. We did not have access to a $B$-glucan assay during our study. It has been suggested that a combination of high levels of B-glucan and the $C$. albicans germ tube antibody can differentiate between Candida colonization and invasive candidiasis in ICU patients with severe abdominal conditions [29]. In the latter study, fewer than $50 \%$ of the patients had CAIs. Furthermore, the study was designed to assess the course of candidiasis in the ICU, rather than to predict the presence of candidiasis on admission. 
A worse outcome associated with YP culture of the peritoneal fluid was evidenced in this study. Furthermore, it was independently associated with mortality. It is the first report in the literature of such impact in mild to moderate infections.

Our study had some potential limitations. First, this was a single-center study. Nevertheless, our center is a large tertiary-care hospital with an experienced, trained team for the care of patients with complicated intra-abdominal infections. Furthermore, peritoneal fluid samples were available for all patients and all were sent for microbiological and mycological culture. However, the present study's results must be validated in multicenter trials. Recently, the sensitivity breakpoints for Candida spp. were modified according to the species [30]. This study used previously described breakpoints $\left(>32 \mu \mathrm{g} \cdot \mathrm{ml}^{-1}\right)$ that could have underestimated the rate of strains resistant to fluconazole. Our study focused on the development of a predictive score and thus did not address the question of how best to treat IAC. It was only associated with increased morbidity and mortality in our population. The comparison of mortality rates according to treatment or not in the group of patients with IAC is not very relevant due to many confounding factors and a clear lack of power. The American guidelines for the treatment of abdominal candidiasis are essentially derived from candidemia guidelines [6]. Recently, an Italian group drew up a list of recommendations for the treatment of IAC [5]. However, in view of the lack of scientific evidence, the vast majority of guidelines are based on expert opinion or extrapolation of data on candidemia [8]. Lastly, the use of the new score is not well calibrated for critical care patients because only one-third of the cohort was admitted to the ICU.

\section{Conclusions}

In conclusion, a score for predicting IAC was respectively built and validated in retrospective and prospective cohorts (featuring a total of 442 CNPIAIs). The new score has a high NPV (for ruling out the need for antifungal treatment at the bedside). It remains to be validated in larger, multicenter cohorts of patients.

\section{Key messages}

- A simple clinical score at bedside may help to avoid antifungal treatment in patients with CNPIAIs.

- The clinical score includes per-operative cardiovascular failure (1 point), upper gastrointestinal tract location of the perforation (1 point), length of stay before surgery more than 48 hours (1 point) and generalized peritonitis (2 points)

- Yeast isolation in patients with CNPIAIs is associated with increased morbidity and independently with increased mortality.

\section{Abbreviations}

APACHE II: Acute Physiology and Chronic Health Evaluation II; AUC: area under the curve; CAl: community-acquired infection; Cl: confidence interval; CNPIAI: complicated non-postoperative intra-abdominal infection; IAC: intra-abdominal candidiasis; ICU: intensive care unit; NPNIAI: nonpostoperative nosocomial intra-abdominal infection; NPV: negative predictive value; OR: odds ratio; PC: prospective cohort; PPV: positive predictive value; RC: retrospective cohort; ROC: receiver operating characteristics; SAPS II: Simplified Acute Physiology Score II; SD: standard deviation; SOFA: Sepsis-related Organ Failure Assessment; YN: yeast negative; YP: yeast positive.

\section{Competing interests}

The authors declare that they have no competing interests.

\section{Authors' contributions}

HD designed the study, participated in data acquisition and analyses, and wrote the manuscript. MG and EZ participated in data acquisition, data analyses, and preparation of the manuscript. AN, MP, SP, JMR, TC and YM participated in the data analyses and drafting of the manuscript. All authors read and approved the manuscript.

\section{Funding}

This research received no specific grant from any funding agency in the public, commercial or not-for-profit sectors. It was funded by the Department of Anesthesiology and Critical Care Medicine of Amiens, University of Picardy Jules Verne. The Department of Anesthesiology and Critical Care Medicine has played no role in the design, in the collection, analysis, and interpretation of data; in the writing of the manuscript; and in the decision to submit the manuscript for publication.

\section{Author details}

'Department of Anesthesiology and Critical Care Medicine, Amiens University Medical Center, 80054 Amiens, Cedex 1, France. ${ }^{2}$ INSERM U1088, Jules Verne University of Picardy, Chemin du Thil, 80039 Amiens, Cedex 1, France. ${ }^{3}$ Digestive and Metabolic Surgery Department, Amiens University Medical Center, 80054 Amiens, Cedex 1, France. ${ }^{4}$ Mycology Laboratory, Amiens University Medical Center, 80054 Amiens, Cedex 1, France.

Received: 17 November 2014 Accepted: 5 February 2015

Published online: 27 February 2015

\section{References}

1. Lee SC, Fung CP, Chen HY, Li CT, Jwo SC, Hung YB, et al. Candida peritonitis due to peptic ulcer perforation: incidence rate, risk factors, prognosis and susceptibility to fluconazole and amphotericin B. Diagn Microbiol Infect Dis. 2002:44:23-7.

2. Montravers P, Chalfine A, Gauzit R, Lepape A, Pierre Marmuse J, Vouillot C, et al. Clinical and therapeutic features of nonpostoperative nosocomial intra-abdominal infections. Ann Surg. 2004;239:409-16.

3. Montravers P, Dupont H, Gauzit R, Veber B, Auboyer C, Blin P, et al. Candida as a risk factor for mortality in peritonitis. Crit Care Med. 2006;34:646-52.

4. Dupont H, Paugam-Burtz C, Muller-Serieys C, Fierobe L, Chosidow D, Marmuse $\mathrm{JP}$, et al. Predictive factors of mortality due to polymicrobial peritonitis with Candida isolation in peritoneal fluid in critically ill patients. Arch Surg. 2002;137:1341-6.

5. Bassetti M, Marchetti M, Chakrabarti A, Colizza S, Garnacho-Montero J, Kett DH, et al. A research agenda on the management of intra-abdominal candidiasis: results from a consensus of multinational experts. Intensive Care Med. 2013:39:2092-106.

6. Solomkin JS, Mazuski JE, Bradley JS, Rodvold KA, Goldstein EJ, Baron EJ, et al. Diagnosis and management of complicated intra-abdominal infection in adults and children: guidelines by the Surgical Infection Society and the Infectious Diseases Society of America. Clin Infect Dis. 2010;50:133-64.

7. Montravers P, Mira JP, Gangneux JP, Leroy O, Lortholary O, for the AmarCand study group. A multicentre study of antifungal strategies and outcome of Candida spp. peritonitis in intensive-care units. Clin Microbiol Infect. 2011;17:1061-7. 
8. Montravers P, Dupont H, Eggimann P. Intra-abdominal candidiasis: the guidelines-forgotten non-candidemic invasive candidiasis. Intensive Care Med. 2013;39:2226-30.

9. Pittet D, Monod M, Suter PM, Frenk E, Auckenthaler R. Candida colonization and subsequent infections in critically ill surgical patients. Ann Surg. 1994;220:751-8.

10. Leon C, Ruiz-Santana S, Saavedra P, Almirante B, Nolla-Salas J, Alvarez-Lerma F, et al. A bedside scoring system ("Candida score") for early antifungal treatment in nonneutropenic critically ill patients with Candida colonization. Crit Care Med. 2006:34:730-7.

11. Ostrosky-Zeichner L, Sable C, Sobel J, Alexander BD, Donowitz G, Kan V, et al. Multicenter retrospective development and validation of a clinical prediction rule for nosocomial invasive candidiasis in the intensive care setting. Eur J Clin Mircrobiol Infect Dis. 2007;26:271-6.

12. Leon C, Ruiz-Santana S, Saavedra P, Galvan B, Blanco A, Castro C, et al. Usefulness of the "Candida score" for discriminating between Candida colonization and invasive candidiasis in non-neutropenic critically ill patients: a prospective multicenter study. Crit Care Med. 2009;37:1624-33.

13. Dupont H, Bourichon A, Paugam-Burtz C, Mantz J, Desmonts JM. Can yeast isolation in peritoneal fluid be predicted in intensive care unit patients with peritonitis? Crit Care Med. 2003;31:752-7.

14. Paphitou NI, Ostrosky-Zeichner L, Rex JH. Rules for identifying patients at increased risk for candidal infections in the surgical intensive care unit: approach to developing practical criteria for systematic use in antifungal prophylaxis trials. Med Mycol. 2005;43:235-43.

15. Bosscha K, van Vroonhoven TJ, van der Werken C. Surgical management of severe secondary peritonitis. Br J Surg. 1999;86:1371-7.

16. Owens WD, Felts JA, Spitznagel EL. ASA physical status classifications: a study of consistency of ratings. Anesthesiology. 1978;49:239-43.

17. Linder MM, Wacha H, Feldmann U, Wesch G, Streifensand RA, Gundlach E. The Mannheim peritonitis index. An instrument for the intraoperative prognosis of peritonitis. Chirurg. 1987;58:84-92.

18. Knaus WA, Draper EA, Wagner DP, Zimmerman JE. APACHE II: a severity of disease classification system. Crit Care Med. 1985;13:818-29.

19. Le Gall JR, Lemeshow S, Saulnier F. A new simplified acute physiology score (SAPS II) based on a European-North American multicenter study. JAMA 1993;270:29057-68.

20. Vincent JL, Moreno R, Takala J, Willatts S, De Mendonca A, Bruining H, et al. The SOFA (Sepsis-related Organ Failure Assessment) score to describe organ dysfunction/failure. On behalf of the Working Group on Sepsis-Related Problems of the European Society of Intensive Care Medicine. Intensive Care Med. 1996:22:707-10

21. Peng $C$, Lee $K$, Ingersoll $G$. An introduction to logistic regression analysis and reporting. J Educ Res. 2002;96:1-14.

22. Hanley J. Receiver operating characteristics (ROC) methodology: the state of the art. Crit Rev Diagn Imaging. 1989;29:307-35.

23. Riche FC, Dray X, Laisne MJ, Mateo J, Raskine L, Sanson-Le Pors MJ, et al. Factors associated with septic shock and mortality in generalized peritonitis: comparison between community-acquired and postoperative peritonitis. Crit Care. 2009;13:R99.

24. Shan YS, Hsu HP, Hsieh YH, Sy ED, Lee JC, Lin PW. Significance of intraoperative peritoneal culture of fungus in perforated peptic ulcer. Br J Surg. 2003;90:1215-9.

25. Hernandez MJ, Balaguer J, Armero R, Baldo J, Gomez L, Solaz C. Candida peritonitis: prevalence and risk factors. Rev Iberoam Micol. 2013;30:189-92.

26. Eggimann P, Garbino J, Pittet D. Epidemiology of Candida species infections in critically ill non-immunosuppressed patients. Lancet Infect Dis. 2003;3:685-702.

27. Lortholary O, Desnos-Ollivier M, Sitbon K, Fontanet A, Bretagne S, Dromer F, et al. Recent exposure to caspofungin or fluconazole influences the epidemiology of candidemia: a prospective multicenter study involving 2,441 patients. Antimicrob Agents Chemother. 2011;55:532-8.

28. Tissot F, Lamoth F, Hauser PM, Orasch C, Fluckiger U, Siegemund M, et al. Beta-glucan antigenemia anticipates diagnosis of blood culture-negative intra-abdominal candidiasis. Am J Respir Crit Care Med. 2013;188:1100-9.
29. Leon C, Ruiz-Santana S, Saavedra P, Castro C, Ubeda A, Loza A, et al. Value of beta-D-glucan and Candida albicans germ tube antibody for discriminating between Candida colonization and invasive candidiasis in patients with severe abdominal conditions. Intensive Care Med. 2012;38:1315-25.

30. Arendrup MC, Cuenca-Estrella M, Lass-Flörl C, Hope WW. EUCAST technical note on Candida and micafungin, anidulafungin and fluconazole. Clin Mycoses. 2014;57:377-9.

\section{Submit your next manuscript to BioMed Central and take full advantage of:}

- Convenient online submission

- Thorough peer review

- No space constraints or color figure charges

- Immediate publication on acceptance

- Inclusion in PubMed, CAS, Scopus and Google Scholar

- Research which is freely available for redistribution 